\title{
The effects of therapeutic group singing on voice, cough and quality of life in parkinson's disease
}

\author{
Cecilia Brooks², Danielle Porter², Daniel Furnas ${ }^{1}$, Judith Maige Wingate ${ }^{1}$ \\ 'Department of Communication Sciences \& Disorders, Jacksonville University, Jacksonville, FL; ${ }^{2}$ Brooks Rehabilitation, Jacksonville, FL, United States
}

Purpose: To examine the effect of a group therapeutic singing intervention on voice, cough, and quality of life in persons with Parkinson Disease (PD) in a community-based outpatient setting using a repeated measures design.

Methods: 19 volunteer participants with PD completed the study. Ten participants participated in the intervention and nine served voluntarily as controls. Participants completed one hour group singing sessions over 12 weeks led by a music therapist. Sessions consisted of 30 min of high intensity vocal exercise and 15 to 20 minutes of group singing. Data on phonation, speech, cough, and quality of life were collected pre-intervention and one week post intervention with final data collection 12 weeks post-intervention.

Results: No significant change in voice measures although $50 \%$ of participants showed improvement. A main effect was found for breathiness $(p=0.023)$, appropriate pitch level $(p=0.037)$ and speaking rate $(p=0.009)$. No main effect for cough but pairwise comparisons were nearly significant pre to post intervention $(p=0.053)$ and pre-intervention to final follow up ( $p=0.023$ ). No main effect found for QOL but singing participants demonstrated better QOL scores than controls.

Conclusions: Results from this small sample suggest that there are some speech benefits from singing intervention as well as potential improvement in cough for airway clearance. Additional study is needed to confirm these results.

Keywords: Cough, Parkinson Disease, Singing, voice

\section{INTRODUCTION}

For people with Parkinson's disease (PD), secondary motor symptoms such as impaired swallow and cough are major contributors to mortality [1-4]. Nearly $90 \%$ of people with PD will develop speech and/or swallowing disorders during the course of the disease [5-7] which can have a negative impact on functional communication and swallowing contributing to a decrease in quality of life for people with PD and their families.

The importance of cough for clearing foreign particles from the airway cannot be overstated for persons with PD as they are at great risk for penetration/aspiration of food and liquids into the lungs [4,8-10]. Cough is a mechanism of airway clearance and is comprised of 3 components: 1 ) inspiratory effort involving chest wall and laryngeal inspiratory muscles, 2) compression of the vocal folds with rapid vocal fold adduction due to contraction of thyroarytenoid and interarytenoid muscles, and 3) finally, contraction of the expiratory muscles with the majority of force produced by the internal

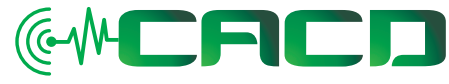

Received: April 29, 2021

Revision: August 30, 2021

Accepted: August 30, 2021

Correspondence:

Judith Maige Wingate

Department of Communication Sciences \& Disorders, Jacksonville University, 2800 University Blvd. N, Jacksonville, FL 32211, United States

Tel: +904-256-8912

Fax: +9042567544

E-mail: jwingat2@ju.edu

(C) 2021 The Korean Association of SpeechLanguage Pathologists

This is an Open Access article distributed under the terms of the Creative Commons Attribution NonCommercial License (https://creativecommons.org/ licenses/by-nc/4.0/) which permits unrestricted noncommercial use, distribution, and reproduction in any medium, provided the original work is properly cited. 
and external oblique muscles $[4,10,11]$. The generation of high-airflow expiratory velocity provides enough force to remove any aspirated particles from the airway $[3,8]$. Ebihara and associates [3] used cough peak flow to measure the motor component of cough in 25 female patients with idiopathic PD. They found the intensity of maximal voluntary cough in patients with both early and advanced PD was significantly weaker than the cough intensity of control subjects. Additional research provides strong evidence that cough function is reduced with neurologic and neurodegenerative diseases and that disordered cough should be expected to occur with disordered swallowing $[2-4,8,9]$.

Singing, like speech, is produced by the larynx in combination with the respiratory system. Singing recruits many of the same muscles associated with swallowing and may increase laryngeal and respiratory muscle strength needed to delay the impending swallowing and respiratory complications due to muscle weakness associated with PD. During singing there is increased neural activation of the same structures and mechanisms used for speech $[5,6]$. Therapeutic singing intervention has been used in previous studies for persons with PD [12-19] and results have demonstrated significant improvements in vocal duration, intonation, maximum inspiratory and expiratory pressure, swallow function and quality of life. Singing has also been shown to promote louder voice production than typical speech and may assist patients in learning to develop and train their respiratory sufficiency $[5,14,15]$. Interventions focused on singing may provide additional benefits other than improved voicing and sustained phonation because of the increased emphasis on the respiratory muscle control system $[4,5,13]$. A study by Di Benedetto's group [16] demonstrated significant pre- and post-treatment differences in 20 subjects with PD who participated in choral singing treatment including improvements in functional residual capacity, maximum inspiratory pressure, maximum expiratory pressure and maximum phonation time. Stegemoller et al. [17] established that after eight weeks of singing training, participants with PD demonstrated significant improvements in breath control and swallow. In addition, there are cognitive and social benefits that occur with group singing $[5,13,14]$. Several of these studies compared differences in voice, swallow and respiration in PD groups that sing to those who do not sing. To date, however, there have not been any studies that have examined changes in cough strength following a singing intervention. Thus, the purpose of this study is to examine the effects of a group therapeutic singing intervention on voice, speech, cough and quality of life (QOL) in individuals with PD compared to a control group. We examined measures of voice, cough and QOL in individuals with PD. We hypothesized that those individuals with PD who participated in the singing group would demonstrate improved performance on all voice, speech, cough and quality of life outcome measures due to weekly engagement in singing. We also hypothesized that the singing group would show continued improvement on all voice and cough outcome measures 3 months post singing intervention compared to the control group.

\section{METHODS}

\section{Participants}

This study was a prospective, complete repeated measures design. After obtaining approval from the university's Institutional Review Board, 26 individuals with Parkinson's disease were recruited for the study. Four recruits did not meet inclusion criteria. Twenty-two participants were enrolled, but only 19 completed the full study, 10 males and 9 females. They were recruited from local PD support groups \& PD exercise/ wellness classes, and none of the participants were actively enrolled in speech therapy. Participants were allowed to choose to participate in either the singing group or the control group to minimize attrition with 9 of the 19 electing to serve as controls. At the time of enrollment, controls indicated their understanding that they would not receive the group singing treatment. Participants ranged in age from 52-79 years with a median age of 68 years for the intervention group and 69 years for the control group. Table 1 contains further participant information. Inclusion criteria were diagnosis of mild to moderate PD as determined by Hoehn and Yahr stages [20] and stable PD medication for at least 30 days prior to enrollment. Exclusion criteria included significant cognitive impairment as demonstrated by a Mini-Mental State Exam (MMSE) score $<24$ [21], depression score of greater than 18 on the Beck Depression Inventory [22], history of smoking in the last 5 years, history of head or neck cancer, asthma, COPD or other neurological disorders and untreated hypertension. All participants gave written informed consent, and the study procedures were approved by the university's Institutional Review Board.

\section{Funding}

Monetary support was provided by a rehabilitation hospital fund to encourage clinician research. The funds were used for supplies, to support the music therapist's time, and to reim- 
Table 1. Demographic information for participants and controls

\begin{tabular}{lcc}
\hline & Participants & Controls \\
\hline Number & 10 & 9 \\
Gender & 5 female & 4 female \\
& 5 male & 5 male \\
Median age & 68 years & 69 years \\
Median MMSE score & 27.67 & 28.28 \\
Median BDI score & 6.33 & 7.57 \\
\hline
\end{tabular}

burse time for university faculty assisting with data collection and analysis. The fundor does not retain control over the design or dissemination of the results of this study.

\section{Singing Intervention}

The singing group regularly attended weekly one-hour group singing sessions for 12 weeks, led by a board-certified music therapist. Each session began with vocal exercises followed by group singing of familiar songs such as Home on the Range [23], You are My Sunshine [24], and Take me Home, Country Roads [25]. Short rest breaks were interspersed throughout the hour session with exercises lasting about 30 minutes. Exercises included the following.

1. Lip buzzing/lip trills. Lip buzzes were performed with no voice then voiced with low, medium, and high frequencies of each participant's comfort level. Participants were encouraged to change pitch levels while maintaining lip buzzing. This exercise targeted respiratory control.

2. Glissandos. Participants sang intervals, starting with a third and moving to octave leaps. This exercise targeted frequency range.

3. Mezze de voce. The group sang vowels, starting off softly, gradually increasing to loud levels, and then back to soft volume. Participants were encouraged to complete the task on a single breath. This exercise targeted intensity control.

4. Articulation exercises. Participants sang consonant-vowel syllables on 5 note patterns at varying speeds. This exercise targeted articulatory precision.

5. Group singing. Participants sang together for 15 to 20 minutes. Familiar songs were used. This exercise targeted controlling phrase length and articulatory clarity.

\section{Data collection}

This study utilized a repeated measures design to test the hypothesis that participants in the singing group would show greater changes in voice, speech, cough and quality of life measures. Participants in both the intervention and the control groups completed a baseline assessment prior to initiation of the singing intervention and repeated the same assessment at one-week post-intervention and again at 12-week post-intervention to examine maintenance of effects.

Voice measures were collected and analyzed using iPad applications. For phonation duration, each participant was asked to sustain the vowel sounds $/ \alpha /$ and $/ \mathrm{i} /$ for as long as possible at a comfortable intensity level. Duration was measured using the stopwatch feature on the iPad Decibel X meter application for duration from beginning of phonation until completion. For sound pressure level (SPL) each participant was instructed to make the loudest sound possible on a sustained $/ \alpha /$. Vocal intensity was also measured using the same Decibel Meter application. The iPad was placed in the same horizontal plane with the microphone closest to the participant's mouth and $50 \mathrm{~cm}$ in front of the participant's mouth. The same device was used for all data collection and recordings were made in the same environment. Additionally, values from the iPad Decibel X Meter were verified against a sound pressure meter. To assess phonation range, each participant was asked to produce/ $\alpha$ / in an upward and downward pitch glide. The lowest and highest fundamental frequency was recorded in Hertz using the Pitch Analyzer iPad application. On all acoustic measures a minimum of three and maximum of five trials were performed. Measures were averaged together for analysis purposes.

Speech samples of a descriptive task using the picture description from the Western Aphasia Battery [26] were recorded using the iPad Voice Recorder. An expert panel of three Speech-Language Pathologists with over five years of experience in motor speech disorders completed perceptual ratings of the recorded samples. The raters were blinded to participant vs. control status. Perceptual ratings for inflection, rate, articulatory precision and speech intelligibility were completed using a five-point scale, adapted from Skinder-Meredith [27]. See Appendix 1 for Perceptual Rating Scale. Words per minute were calculated from the recorded samples.

Cough measures were assessed by having participants produce a voluntary cough into a peak flow meter, MicroLife Model PF100, to assess peak flow as measured in liters/minute. Three to five trials were completed for each participant in order to obtain average measures of airflow.

Participant perception of their quality of life related to voice and communication was captured using the Communicative 
Effectiveness Survey- Revised (CES-R) [28] and the Voice Handicap Index (VHI) [29].

\section{RESULTS}

The hypothesis stated that participants in the singing group would show greater improvements on measures of voice, speech, cough and quality of life in comparison to the control group. To test the hypothesis a repeated measures ANCOVA was used with age and gender as covariates throughout. Main effects were tested and comparison of the pre- and post-intervention conditions as well as the maintenance condition were completed.

There were no significant differences between the participant $(\mathrm{N}=12)$ and control groups $(\mathrm{N}=7)$ for age, gender, MMSE and BDI.

\section{Voice measures}

No main effects were found for vowel duration $(\mathrm{F}=1.020, p=$ 0.372 ) or intensity ( $\mathrm{F}=2.74, p=0.083)$. Some participants were below the expected mean duration of 13.5 seconds, (range 8.5-19.5 sec) [32] at baseline but had moved to within expected ranges by the end of the study. Likewise, there was no main effect found for maximum pitch ( $\mathrm{F}=1.207, p=0.313)$ or

Table 2. Changes in frequency range in semitones

\begin{tabular}{|c|c|c|c|c|c|}
\hline $\begin{array}{l}\text { Range } \\
\text { (Semitones) }\end{array}$ & $\begin{array}{c}\text { Pre- } \\
\text { Intervention }\end{array}$ & $\begin{array}{c}\text { Post- } \\
\text { Intervention }\end{array}$ & Change & Maintenance & $\begin{array}{l}\text { Overall } \\
\text { change }\end{array}$ \\
\hline \multirow[t]{10}{*}{ Participants } & 16 & 13 & -3 & 16 & 0 \\
\hline & 13 & 6 & -7 & 18 & 5 \\
\hline & 13 & 9 & -4 & 23 & 10 \\
\hline & 23 & 17 & -6 & 17 & -6 \\
\hline & 13 & 10 & -3 & 18 & 5 \\
\hline & 10 & 10 & 0 & 7 & -3 \\
\hline & 19 & 16 & -3 & 17 & -2 \\
\hline & 16 & 24 & 8 & 8 & -8 \\
\hline & 22 & 22 & 0 & 19 & -3 \\
\hline & 13 & 18 & 5 & 16 & 3 \\
\hline \multicolumn{6}{|l|}{ Controls } \\
\hline & 25 & 23 & -2 & 20 & -5 \\
\hline & 23 & 31 & 8 & 27 & 4 \\
\hline & 4 & 9 & 5 & 10 & 6 \\
\hline & 18 & 5 & -7 & 12 & -6 \\
\hline & 11 & 12 & 1 & 13 & 2 \\
\hline & 18 & 13 & -5 & 15 & -3 \\
\hline & 21 & 25 & 4 & 11 & -10 \\
\hline & 14 & 12 & -2 & 12 & -2 \\
\hline
\end{tabular}

minimum pitch ( $\mathrm{F}=1.68, p=0.203)$. Pitch ranges were calculated in semitones to more easily examine the relative change across participants. Over half of the active participants (55\%) showed some improvement in range (3-10 semitones) or maintained the same range, with $45 \%$ showing a decline in range (2 to 8 semitones). Forty-four percent of controls showed a positive change in range (2-6 semitones) with $56 \%$ showing a decrease ranging from 2 to 10 semitones. Individual results are contained In Table 2.

\section{Speech measures}

A number of perceptual ratings were made by 3 experienced raters. Their ratings were averaged together for each data collection point. Main effects were found for breathiness/weakness by age ( $\mathrm{F}=5.231, p=0.023)$ and for appropriate pitch level by gender ( $\mathrm{F}=5.231, p=0.037)$. The breathiness ratings were markedly higher in the 71-80 years age range as compared to participants 50-70 years of age. For appropriate pitch ratings, females stayed at the same rating post-training (50\%) or improved (50\%) while the perception of pitch declined in $33 \%$ of the male participants and $66 \%$ displayed improved ratings. No main effects were found for the other perceptual ratings (Table 3 ).

A main effect of treatment was found for speech rate as calculated in words per minute $(\mathrm{F}=5.501, p=0.009)$. See individual results in Figures 1 and 2.

\section{Cough measure}

No main effect was found for cough ( $\mathrm{F}=3.154, p=0.057)$. However, pairwise comparisons within the treatment group were nearly significant between pre- and post-testing ( $p=$

Table 3. Perceptual ratings

\begin{tabular}{ll}
\hline Parameter & \multicolumn{1}{c}{ Statistic } \\
\hline Appropriate prosody & $\mathrm{F}=.255, p=0.776$ \\
Hoarseness & $\mathrm{F}=.442, p=0.647$ \\
Breathiness/weakness & $\mathrm{F}=.567, p=0.246$ \\
Appropriate pitch level & $\mathrm{F}=.067, p=0.935$ \\
Appropriate loudness & $\mathrm{F}=.219, p=0.804$ \\
Loudness decay & $\mathrm{F}=2.525, p=0.097$ \\
Consistent rate & $\mathrm{F}=.950, p=0.398$ \\
Appropriate rate & $\mathrm{F}=2.766, p=0.079$ \\
Precision of consonants & $\mathrm{F}=1.788 . p=0.185$ \\
Intelligibility of speech & $\mathrm{F}=1.247, p=0.302$ \\
\hline
\end{tabular}




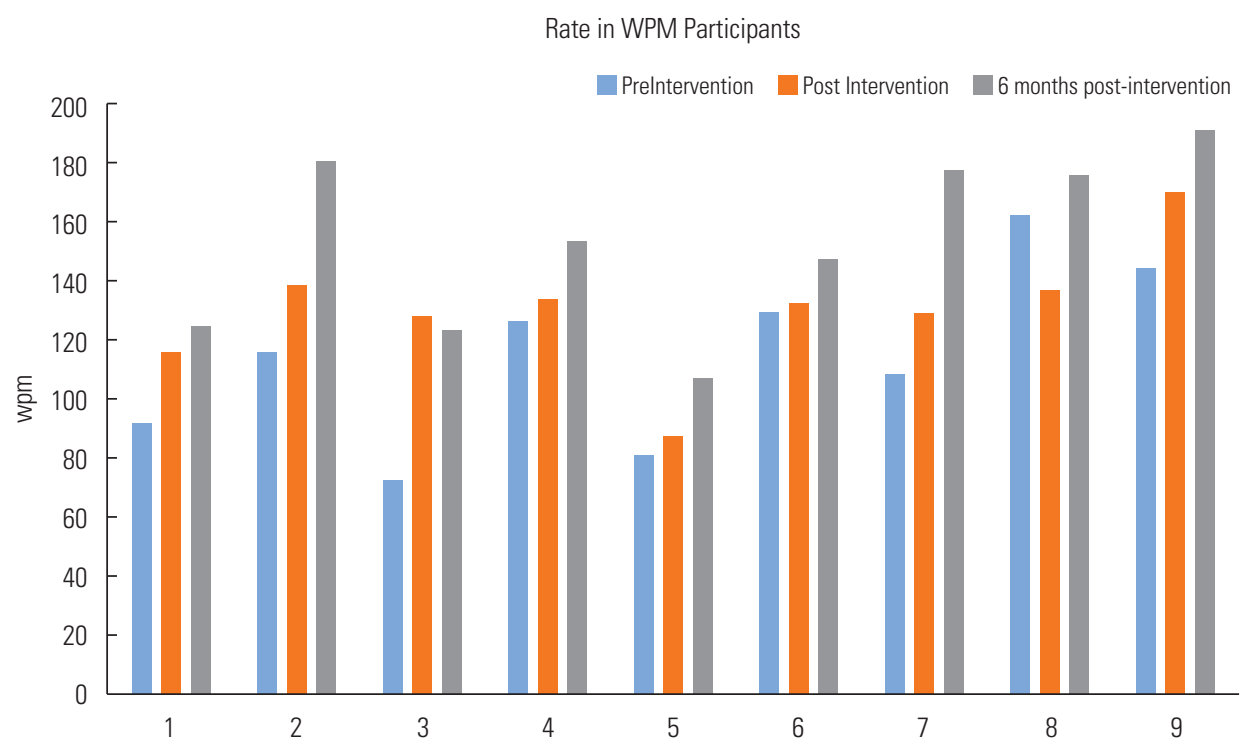

Figure 1. Speaking rate for singing group participants.

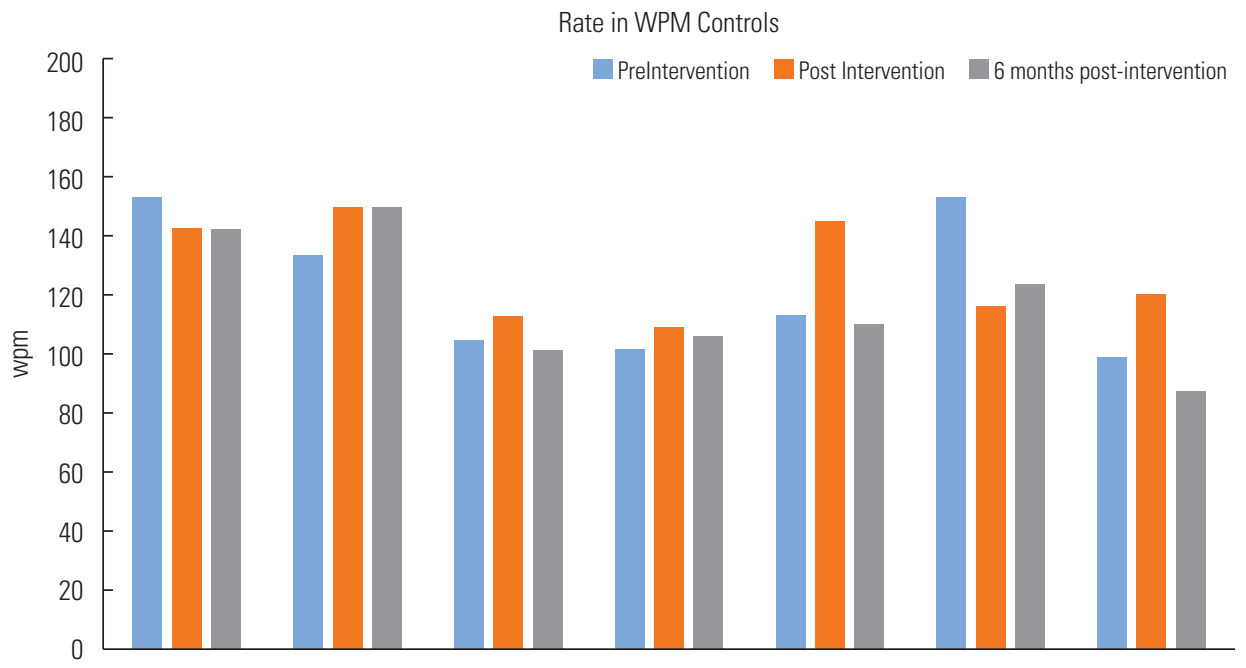

Figure 2. Speaking rate for controls.

0.053) and between pre-testing and the maintenance measurement point ( $p=0.023$ ) (Figures 3 and 4 ).

\section{Quality of life measures}

No main effects were found on the two quality of life measures, the VHI $(\mathrm{F}=.193, p=0.826)$ and the CES $(\mathrm{F}=2.553, p=0.095)$, both self-rated scales. Mean scores were compared for participants and controls. The singing group displayed lower VHI scores across time than did the control group indicating more positive quality of life (Figures 5 and 6). For the CES, for which a higher score is more desirable, the singing group participants demonstrated higher scores than the control group al- though the change in scores was not significant.

\section{DISCUSSION}

The purpose of this pilot study was to investigate the effects of choral singing on speech, voice, cough and quality of life in people with idiopathic PD. To our knowledge this is the first study to measure peak flow during voluntary cough as a component of cough strength following a choral singing intervention for persons with PD. Peak expiratory flow is one component of cough and was the measure utilized for this study. For the majority of participants $(60 \%)$, baseline measures were 


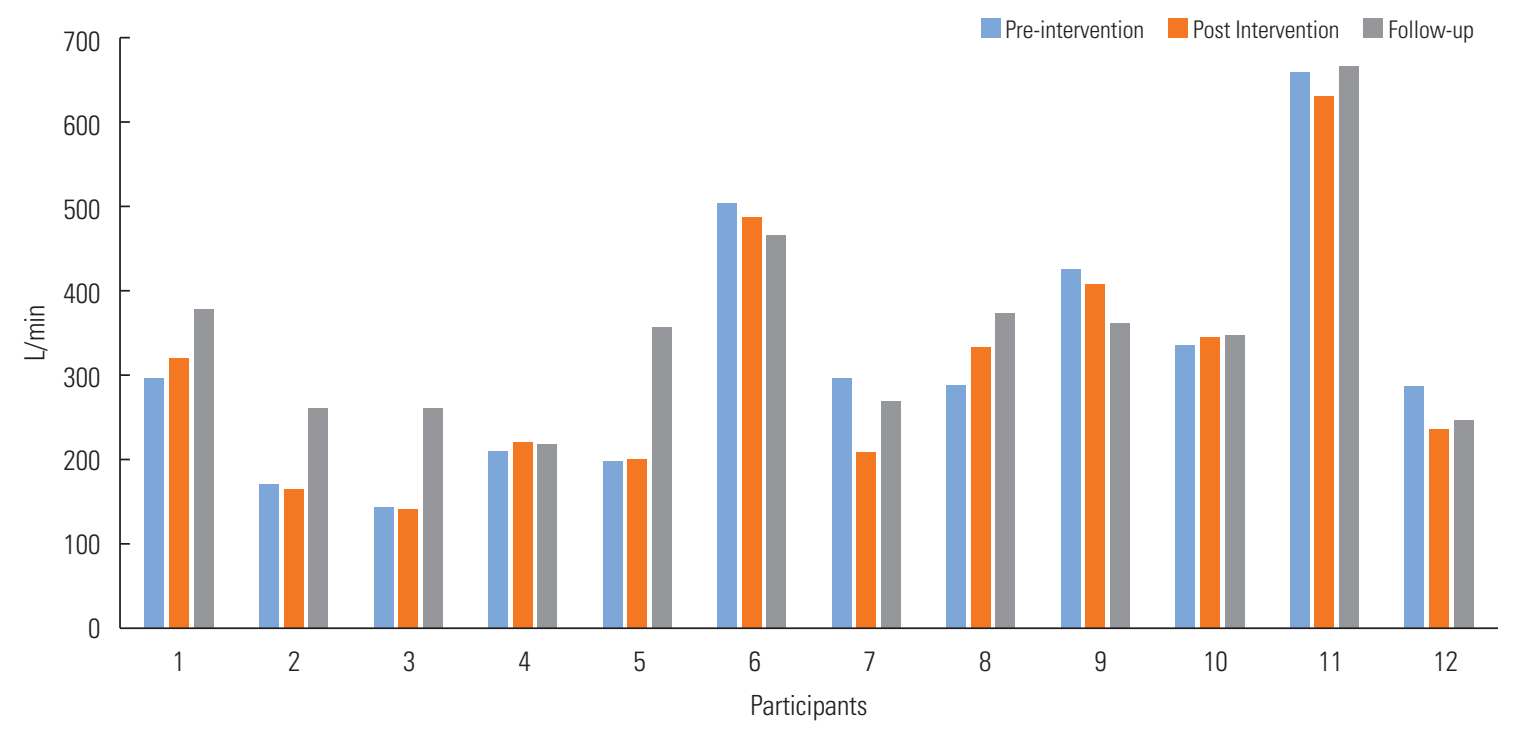

Figure 3. Peak expiratory flow for singing participants.

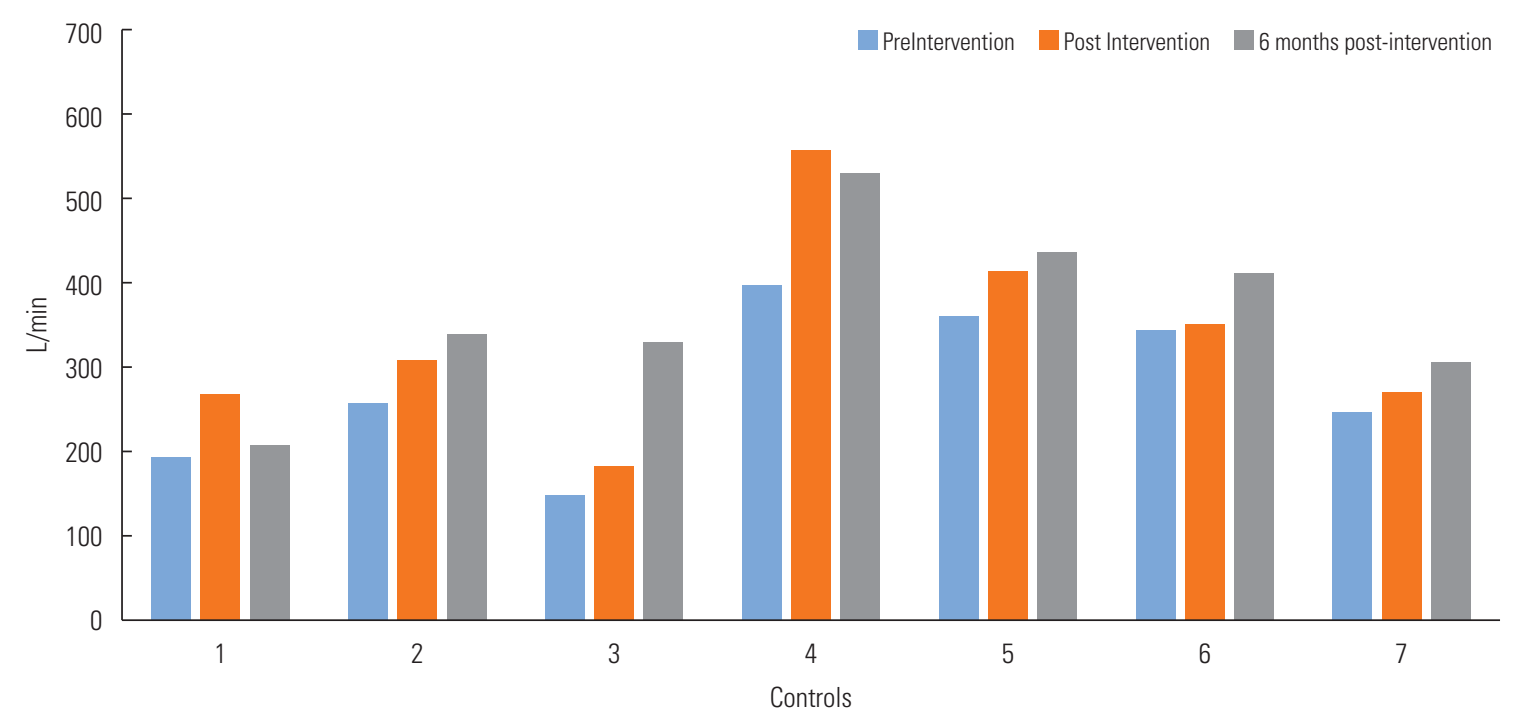

Figure 4. Peak expiratory flow for controls.

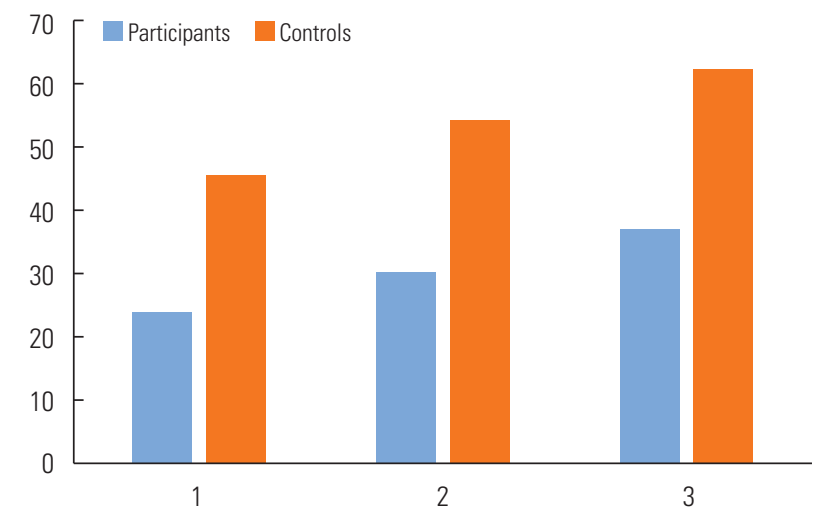

Figure 5. Mean VHI scores.

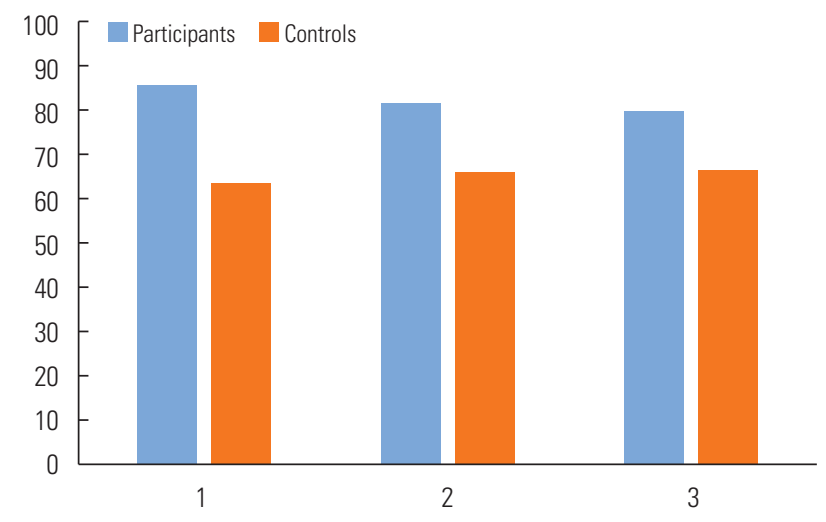

Figure 6. Mean CES scores. 
below the expected averages of 300 to $500 \mathrm{~L} / \mathrm{min}$ for older adults. Following the singing intervention, $70 \%$ of participants were within the expected range while only $55 \%$ of the controls were in the expected range. This improvement, while not statistically significant, shows clinical importance. The emphasis on respiratory control by engaging abdominal muscles in singing activities may contribute to improved peak expiratory flow for cough. It should be noted that many of the participants, as well as the controls, were very physically active in a PD-focused exercise group, despite not being actively enrolled in speech/voice therapy during the study. This increased physical activity could limit the nature of the changes seen within respiratory control and cough compared to more sedentary adults with PD.

Maximum vowel duration did not change significantly posttraining, although some participants did display improved values to within expected ranges for their age following training. Likewise, participants did not show a significant change in intensity levels post-training. This may represent a ceiling effect as most participants were able to produce between 70 and $90 \mathrm{~dB}$ when asked to make sound as loudly as they could. While no main effect was found for frequency range, $55 \%$ of singing participants showed a positive increase in range compared to $44 \%$ of the controls. This suggests that the singing exercises had a positive effect on frequency range despite not reaching statistical significance.

The participants in this study had mild to moderate Parkinson's Disease and exhibited very few speech complaints. As a result, few significant changes were found in the speech measures, likely representing a ceiling effect. The perception of breathiness was greater in participants over the age of 70 . This is likely a result of an aging effect. Some of the participants may have had bowed vocal folds or a vocal fold paresis or paralysis that was undiagnosed as they did not have a laryngoscopy performed prior to the start of the study. One-third of the male participants showed a decline in appropriate pitch levels during a picture description task. This may represent a gender difference as men may have significantly more speech problems from PD than women [31].

Treatment group participants showed a significant improvement in speaking rate during a picture description task. This occurred without a decrease in perceived intelligibility or change in perceived appropriateness of rate. There were no changes noted in short rushes of speech. Therefore, this increased speech rate could be a result of the articulatory and respiratory control exercises performed during the intervention.
The investigators expected to see improvements in quality of life (QOL) as measured by the Voice Handicap Index and the Communication Effectiveness survey but this was not the case. A significant change on the VHI is 18 points [29]. Half of the participants showed a greater than 18 point decline in QOL score at the maintenance point. At least one previous study [13] found an increase in quality of life scores posttraining. However, a study by Shih and colleagues [12] which also used a 12 week singing intervention, found no change in quality of life scores using the Voice Handicap Index post-intervention. Other studies have found a decline in QOL scores after singing intervention or as a result of disease progression so this may have impacted the current result [32,33]. Further, people with PD may have sensory deficits that may compromise perceptual judgement. Several studies suggest that there are difficulties in persons with PD in terms of perceptual estimates involving vision and motor planning $[34,35]$. This could suggest similar issues with perception through other mechanisms.

\section{Limitations}

The study size (10 participants and 9 controls) is small and limits the conclusions that can be drawn from the results of this pilot study. However, the number of participants in similar studies have been in the 9 to 24 range. The study was conducted in a large metropolitan area with limited public transportation so the ability of potential participants to travel to the meeting site weekly likely limited participation as well as the variability of participants. Most of the participants displayed mild to moderate symptoms of PD contributing to ceiling effects with some parameters. Participants and controls in this study were highly active which may have further contributed to a ceiling effect. Further, our final measures were taken during the beginning of the holiday season which could have contributed to the negative changes seen in the quality of life measures.

Only one component of cough was measured. Peak flow, while an important cough component, is inter-related with vocal fold compression and inspiratory volume. Given the increased breathiness measures in participants over 70 , vocal fold thinning, bowing or paralysis may have limited compression, which in-turn limits cough efficacy. Further investigation using full spirometric data is warranted to fully evaluate changes in cough and respiration following a singing intervention. 


\section{CONCLUSIONS}

This study is the first to suggest that enhanced airway clearance via cough may be improved through a group singing intervention while providing other positive effects on voice and speech parameters. A singing intervention is low-cost and provides social interaction for persons with $\mathrm{PD}$ and can easily occur in community settings. Further investigation of singing intervention and its effect on cough should be completed using full spirometric measures.

\section{REFERENCES}

1. Fall PA, Saleh A, Fredrickson M, Olsson JE, Granérus AK. Survival time, mortality, and cause of death in elderly patients with Parkinson's disease: a 9-year follow-up. Mov Disor. 2003;18:1212-1216.

2. Troche M., Schumann B, Brandimore A, Okun M, Hegland K. Reflex cough and disease duration as predictors of swallowing dysfunction in Parkinson's disease. Dysphagia. 2016;31:757-764.

3. Ebihara S, Saito H, Kanda A, Nakajoh M, Takahashi H, Arai H, et al. Impaired efficacy of cough in patients with Parkinson's disease. Chest. 2003;124:1009-1015.

4. Pitts T, Bolser D, Rosenbek J, Troche M, Sapienza C. Voluntary cough production and swallow dysfunction in Parkinson's disease. Dysphagia. 2008;23:297-301.

5. Tamplin J, Morris M, Marigliani C, Baker F, Vogel A. ParkinSong: A controlled trial of singing-based therapy for Parkinson's disease. Neurorehabil Neural Repair. 2019;33:453-463.

6. Amorim G, Albuquerque L, Pernambuco L, Balata P, Luckwu-Lucena B, Silva $\mathrm{H}$. Contributions of neuroimaging in singing voice studies: a systematic review. Revista CEFAC. 2071;19:556-564.

7. Dashtipour K, Tafreshi A, Lee J, Crawley B. Speech Disorders in Parkinson's Disease: Pathophysiology, Medical Management and Surgical Approaches. Neurodegener Dis Manag. 2018;8:337-348.

8. Fontana G, Pantaleo T, Lavorinin F, Benvenuti F, Gangemi S. Defective motor control of coughing in Parkinson's disease. Am J Respir Crit Care Med. 1998;158:458-464.

9. Hegland K, Okun M, Troche M. Sequential voluntary cough and aspiration or aspiration risk in Parkinson's disease. Lung. 2014; 192:601-608.

10. Pitts T, Bolser D, Rosenbek J, Troche M, Okun M, Sapienza C. Impact of expiratory muscle strength training on voluntary cough and swallow function in Parkinson disease. Chest. 2009;135:13011308.

11. Mills C, Jones R, Huckabee ML. Measuring Voluntary and Reflexive Cough Strength in Healthy Individuals. Respir Med. 2017;132: 95-101.

12. Shih L, Piel J, Warren A, Kraic, L, Silver A, Vanderhorts V, et al. Singing in groups for Parkinson's disease (SING-PD): a pilot study of group singing therapy for PD related voice/speech Disorders. Parkinsonism Relat Disor. 2012;18:548-552.
13. Stegemoller E, Radig H, Hibbing P, Wingate J, Sapienza C. Effects of singing on voice, respiratory control and quality of life in persons with Parkinson's disease. Disabil Rehabil. 2017;39:594-600.

14. Yinger $\mathrm{O}$, LaPointe $\mathrm{L}$. The effects of participation in a group music therapy voice protocol (G-MTVP) on the speech of individuals with Parkinson's disease. Music Ther Perspect. 2012;30:25-31.

15. Haneishi E. Effects of a music therapy voice protocol on speech intelligibility, vocal acoustic measures, and mood of individuals with Parkinson's disease. J Music Ther. 2001;38:273-290.

16. DiBenedetto P, Cavazzon M, Mondolo F, Rugiu G, Peratoner A, Biasutti E. Voice and choral singing treatment: a new approach for speech and voice disorders in Parkinson's disease. Eur J Phys Rehabil Med. 2009;45:13-19.

17. Stegemoller E, Hibbing P, Radig H, Wingate J. Therapeutic singing as an early intervention for swallowing in persons with Parkinson's disease. Complemen Ther Med. 2017;31:127-133.

18. Elefant C, Baker F, Lotan M, Lagesen S, Skeie G. The effect of group music therapy on mood, speech, and singing in individuals with Parkinson's disease - a feasibility study. J Music Ther. 2012; 49:278-302.

19. Tautscher-Basnett A, Tomantschger V, Keglevic S, Freimuller M. Group therapy for individuals with Parkinson disease (PD) focusing on voice strengthening. Proc 4th World Congr Neurorehabil. 2006;P1-054

20. Hoehn MM, Yahr MD. Parkinsonism: onset, progression and mortality. Neurol. 2001;57:S11-S26.

21. Folstein M, McHugh P. Mini mental state a practical method for grading the cognitive state of patients for the clinician. J Psychiatr Res. 1975;12:189-198.

22. Beck AT, Ward CH, Mendelson M, Mock J, Erbaugh J. An inventory for measuring depression. Arch Gen Psychiatry.1961;4:561-571.

23. Higley B. Home On The Range. 1874; Retrieved from: https:// www.azlyrics.com/lyrics/davidallancoe/homeontherange.html

24. Davis, J, Mitchell, C. You Are My Sunshine. [Recorded by Copeland, 1939]. On The Grey Man EP. Retrieved, from: https://www. azlyrics.com/lyrics/copeland/youaremysunshine.html

25. Denver J, Take Me Home, Country Roads. On Poems, Prayers \& Promises. 1971; Retrieved from: https://www.azlyrics.com/lyrics/ johndenver/takemehomecountryroads.html

26. Shewan CM, Kertexa A. Reliability and validity characteristics of Western Aphasia Battery, J Speech Hear Disor. 1980;45:308-324.

27. Skinder-Meredith AE. Speech characteristics rating form. In C. Bowen, Children's speech sound disorders, 2nd ed. Oxford: WileyBlackwell; 2015. p. 312-318.

28. Donovan N. Examining the item-level psychometric properties of the Communicative Effectiveness Survey- Revised for people with Parkinson's Disease and dysarthria. Clin Arch Comm Disor. 2018; 3:42-51.

29. Jacobson B, Johnson A, Grywalski C, Silbergleit A, Jacobsen G, Benninger M. The Voice Handicap Index (VHI): development and validation. Am J Speech-Lang Pathol. 1997;6:66-70.

30. Kent RD, Kent JF, Rosenbek JC. Maximum performance tests of speech production. J Speech Hear Disor. 1987;52:367-387. 
31. Szewczyk-Krolikowski K, Tomlinson P, Nithi K, Wade-Martins R, Talbot K, Ben-Shlomo Y, et al. The influence of age and gender on motor and non-motor features of early Parkinson's disease: initIal findings from the Oxford Parkinson Disease Center (OPDC) discovery cohort. Parkinsonism Rel Disor. 2014;20:99-105.

32. VanHooren MRA, Baijesn LWJ, Vos R, Pilz W, Kuipers LMF, Kremer B, et al. Voice- and swallow-related quality of life in idiopathic Parkinson's disease. Laryngoscope. 2016;126:408-414.

33. Moreira RC, Zonta MB, deAraujo APS, Israel VL, Teive HAG. Qual- ity of life in Parkinson's disease patients: progression markers of mild to moderate stages. Arq. Neuropsiquiatri. 2017;75:497-502.

34. Martens KAE, Almeida QJ. Dissociating between sensory and perceptual deficits in PD: more than simply a motor deficit. Mov Disor. 2012;27:3887-3892.

35. Silveira CRA, Martens KAE, Pieruccini-Faria F, Bell-Boucher D, Roy EA, Almeida QJ. Disentangling perceptual judgement and online feedback deficits in Parkinson's freezing of gait. J Neurol. 2015;262:1629-1636. 
Appendix 1. Perceptual Rating Scale

\begin{tabular}{|c|c|c|c|c|c|}
\hline & Never & Rarely & Sometimes & Frequently & Always \\
\hline Appropriate prosody & 1 & 2 & 3 & 4 & 5 \\
\hline Hoarse voice & 1 & 2 & 3 & 4 & 5 \\
\hline Breathy/weak voice & 1 & 2 & 3 & 4 & 5 \\
\hline Appropriate pitch level & 1 & 2 & 3 & 4 & 5 \\
\hline Appropriate loudness & 1 & 2 & 3 & 4 & 5 \\
\hline Loudness decay & 1 & 2 & 3 & 4 & 5 \\
\hline Consistent rate (e.g., short rushes of speech) & 1 & 2 & 3 & 4 & 5 \\
\hline Appropriate rate & 1 & 2 & 3 & 4 & 5 \\
\hline Precision of consonants & 1 & 2 & 3 & 4 & 5 \\
\hline Intelligible speech & 1 & 2 & 3 & 4 & 5 \\
\hline
\end{tabular}

Adapted from Skinder-Meredith, 2015. 